\title{
Letter identification errors as a function of retinal input locus and positional variability
}

\author{
FORREST HAUN and W. R. GARNER \\ Yale University, New Haven, Connecticut 06520
}

\begin{abstract}
Retinal acuity differences are shown to account substantially for identification errors resulting when members of a dimensionally defined letter set $(p, b, q, d)$ are singly presented at fixed positions within the foveal field for reduced durations. The error pattern can be altered by shifting the fixation point as little as $12 \mathrm{~min} 9 \mathrm{sec}$ of visual angle. However, when letter position varies, the error distribution reflects dimensional information structure.
\end{abstract}

Receptor organ influences on information processing are so well established in general that their operation in a specific instance is often either simply asserted, or, paradoxically, overlooked in the pursuit of higher order processes. Also well established in information processing is the influence of the informational structure of a stimulus set. But we are just beginning to understand how these two general classes of processing variables-sensory receptor and stimulus informationalinteract. The following experiment addressed that issue by demonstrating that distance from retinal center, and not informational structure, best accounts for errors of letter identification when the letters are visually presented near threshold, even when presentation is entirely within the foveal field. However, when letter position varies, this retinal influence is absent. The latter finding is in contrast to the results of Eriksen and Schultz (1977), who used a reaction time measure of letter identification performance.

The specific letter set used consisted of block $p$, $\mathrm{b}$, $\mathrm{q}$, and $\mathrm{d}$ (see Figure 1), which can be defined as two positions of a square along two dimensions: left or right, up or down about a stem. The set was used in a previous experiment investigating the effects of different types of degradation on letter identification errors (Garner \& Haun, in press). When degradation resulted from reduced presentation duration, more errors occurred along the vertical than the horizontal dimension (i.e., more $\mathrm{p}-\mathrm{b}$ and $\mathrm{q}-\mathrm{d}$ confusions than $\mathrm{p}-\mathrm{q}$ and $b-d$ confusions). This result would not be expected from an informational analysis, given the relative positions of each letter as presented (see Figure 1a). That is, with the center horizontal bar of each letter always appearing in a fixed position, a p-q confusion, for example, involved the misplacement of one line segment while a p-b confusion involved the misplacement of two such segments. So one would expect the former type of error (horizontal) to be morc frequent than the latter type (vertical).

The research reported here was supported by Grant MH 14229 from the National Institute of Mental Health to Yale University.
Three explanations of the obtained result seemed plausible: (1) The ends of the horizontal extent of each letter appeared closer to fixation than the ends of the vertical extent, so that elements permitting horizontal discrimination fell on a more acute portion of the retina than elements permitting vertical discrimination. (2) What actually varied along the two dimensions from presentation to presentation were different forms (the square along the vertical, the vertical bar extending from the square along the horizontal), and if more energy was required to encode the four-line square than the one-line bar, reducing stimulus energy could have had a more disruptive effect on (vertical) square positioning than on (horizontal) bar positioning. (3) More of the $q$ and $d$ were right of fixation, while more of the $p$ and $b$ were left of fixation, so that mere detection of a visual field difference would be sufficient

$\mathbf{a}$
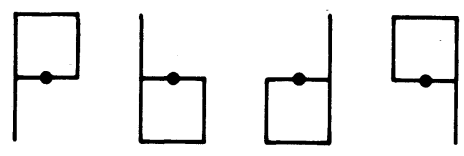

b
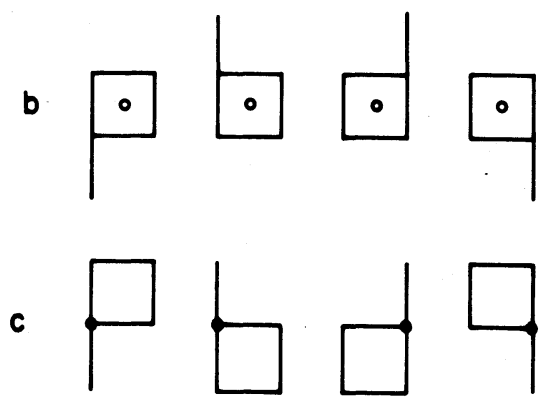

d
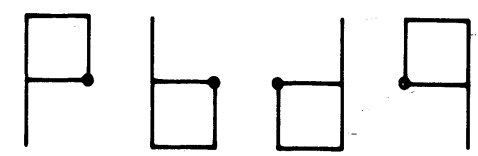

Figure 1. Letter positions vis-a-vis fixation point $(0)$ in four conditions: (a) fixed bar (Garner \& Haun, in press), (b) fixed square, $(c)$ fixed stem, and $(c+d)$ fully variable. 
to yield a horizontal, but not a vertical, dimension discrimination.

These explanations were tested by varying letter position vis-a-vis the fixation point.

\section{METHOD}

\section{Stimuli and Apparatus}

The lowercase letters $p, b, q$, and $d$ were each formed by a Calcomp Pen Plotter using straight black lines $6.35 \mathrm{~mm}$ long and $.6 \mathrm{~mm}$ wide on off-white paper. The sides of the square portion of each letter were composed of one such line, while the stem was formed by adding another of the lines to the left or right side of the square. When affixed to $12.6 \times 17.8 \mathrm{~cm}$ cards and viewed through a Scientific Prototype GB-3 tachistoscope, each letter subtended $17 \mathrm{~min} 11 \mathrm{sec}$ by $34 \mathrm{~min} 23 \mathrm{sec}$ of visual angle. A black fixation dot was placed on another card (white) and positioned in a second field of the tachistoscope. Luminance of both fixation and stimulus fields was set at $6 \mathrm{fL}\left(20.56 \mathrm{~cd} / \mathrm{m}^{2}\right)$, according to coarse lamp controls on the tachistoscope.

\section{Stimulus Conditions}

In each of three conditions, each letter in the set was presented at a different position relative to fixation. These positions are shown in Figure 1 and are described below. The fourth positioning, the fixed bar (Condition a) used in the Garner and Haun (in press) experiment, was not repeated here.

Fixed square (Condition b). One set of letters was positioned such that the square of each appeared in a constant position, with the fixation point at its center. Thus, only the position of the vertical bar varied along both dimensions, permitting both horizontal and vertical information to impinge on the retina equidistant from fixation.

Fixed stem (Condition c). A second letter set was positioned such that the stem of each appeared in a constant position, bisected by the fixation point. Thus, only the position of the square varied along both dimensions; but, in contrast to Condition $b$, the presence of a line segment immediately adjacent to fixation was sufficient to yield horizontal information ( $\mathrm{p}$ p or a b if on the right, a q or a d if on the left), while vertical information required stimulus input appearing $6.35 \mathrm{~mm}$ away from fixation (at either end of the stem, or at the end of the center horizontal).

Fully variable (Condition $\mathrm{c}+\mathrm{d}$ ). A final set of letters was positioned such that each could appear either primarily left or primarily right of fixation. Four letters had their stems in the center of the cards (Condition c), bisected by the fixation point. The same point fell at different parts of the other four letters (Condition d), whose stems were either left or right of fixation. Over all eight letters, then, both horizontal and vertical information yielding one of the four responses was not available from any fixed location. However, within the subset with stems at the center, horizontal information was available immediately left or right of fixation, while vertical information was $6.35 \mathrm{~mm}$ away from fixation, as in the fixed-stem condition. Within the subset of letters whose stems were left or right of fixation, information permitting discrimination along both dimensions was available immediately adjacent to fixation.

\section{Subjects}

Six members of the Yale University community participated as paid subjects in the experiment. All were right-handed and had normal or corrected-to-normal vision.

\section{Procedure}

Subjects were instructed to focus with both eyes on the fixation point as well as possible. Following a ready cue (the sound of the stimulus holder's insertion into the tachistoscope), subjects initiated a single-letter presentation by pressing a handheld microswitch. Each subject received 160 presentations in each of the three stimulus conditions. All six possible orderings of the conditions were used. In each condition, each of the four letters appeared 40 times in random order; in the fully variable condition 20 of these were left and 20 right of fixation, also in random order.

Prior to the start of each condition, approximately 60 practice trials occurred, the letters appearing randomly at gradually decreasing durations until a stimulus duration was reached that yielded three errors in 10 trials. The 160 trials for that condition were then run at that duration for that subject. The entire experiment lasted about $1.5 \mathrm{~h}$, including two 5 -min breaks between conditions.

\section{RESULTS}

Obtained mean error rates were $28.0 \%$ for the fixedsquare condition, $30.4 \%$ for the fixed-stem condition, and $32.7 \%$ for the fully variable condition; the respective mean stimulus durations producing these error rates were $6.5,5.0$, and $6.0 \mathrm{msec}$. Neither total numbers of errors nor stimulus durations were significantly different across conditions, nor was there any consistent rank ordering of conditions in either errors or durations.

Mean number of errors and standard deviations are shown in Table 1. In planned-comparison tests (twotailed), errors along the vertical dimension exceeded those along the horiztonal dimension only in the fixedstem condition $[\mathrm{t}(20)=3.99, \mathrm{p}<.001]$. All subjects committed more vertical than horizontal errors in this condition, none less than $50 \%$ more.

In the fixed-square condition, two subjects also showed this same pattern, as indicated in Table 1. The overall nonsignificance in that condition was thus due to the other four subjects.

In the fully variable condition, the subset of letters with their stems in the center (i.e., positioned identically as those in the fixed-stem condition) produced a nonsignificant difference in number of horizontal and vertical errors $[t(10)=1.55, p>.20]$. The letter subset with stems left or right of center also showed no significant difference in number of horizontal and

Table 1

Mean Errors and Standard Deviations by Type and Presentation Condition for Letter Set p, b, q, d

\begin{tabular}{|c|c|c|c|c|c|c|}
\hline \multirow[b]{3}{*}{ Condition } & \multicolumn{6}{|c|}{ Error Type } \\
\hline & \multicolumn{2}{|c|}{$\begin{array}{l}\text { Horiztonal } \\
(\mathrm{p}-\mathrm{q}, \mathrm{b}-\mathrm{d})\end{array}$} & \multicolumn{2}{|c|}{$\begin{array}{l}\text { Vertical } \\
(p-b, q-d)\end{array}$} & \multicolumn{2}{|c|}{$\begin{array}{l}\text { Diagonal } \\
(\mathrm{p}-\mathrm{d}, \mathrm{q}-\mathrm{b})\end{array}$} \\
\hline & Mean & SD & Mean & SD & Mean & SD \\
\hline Fixed Square & 13.2 & 5.1 & 19.2 & 6.6 & 12.5 & 3.4 \\
\hline Two Subjects & 12.0 & 4.0 & 27.0 & 0.0 & 11.0 & 0.0 \\
\hline Four Subjects & 13.8 & 4.9 & 15.3 & 2.9 & 13.3 & 3.6 \\
\hline Fixed Stem & 12.5 & 1.9 & 25.5 & 8.9 & 10.7 & 3.5 \\
\hline Fully Variable & 20.3 & 4.9 & 21.2 & 3.7 & 10.8 & 4.2 \\
\hline Center Stem & 11.8 & 4.5 & 8.8 & 2.8 & 6.3 & 1.5 \\
\hline Left or Right Stem & 8.5 & 2.7 & 12.3 & 2.4 & 4.5 & 3.2 \\
\hline
\end{tabular}

Note $-N=6$. 
vertical errors, nor were the two subsets significantly different in total number of errors.

Using Duncan's multiple range test, post hoc comparisons of diagonal errors showed them to be less than the number of vertical errors $(p<.01)$ in the fixed-stem condition but not in the fixed-square condition. In neither of these conditions was number of diagonal and horizontal errors significantly different. In the fully variable condition, however, diagonal errors (numbering half those along either dimension singly) were significantly different from both dimensions $(\mathrm{p}<.05)$.

\section{DISCUSSION}

The position along the retina at which letter elements enter the visual system can influence identification errors, even when all elements enter through the fovea. With the letters $p, b, q$, and $d$ each presented for short durations, vertical errors (i.e., $p-b$ and $q-d$ confusions) outnumbered horizontal errors (i.e., $p-q$ and $b-d$ confusions) when fixation was at one of two points: in the middle of the horizontal bar common to all letters (Garner $\&$ Haun, in press) or in the middle of each letter's stem (the present fixed-stem condition). In both cases, information sufficient for a horizontal discrimination appeared closer to fixation than information sufficient for a vertical discrimination. A retinal acuity difference can therefore account for the greater number of the latter type of errors. This difference would be especially evident at low stimulus durations, which were a priori determined for each subject to yield (in this experiment) $30 \%$ errors. In the Garner and Haun (in press) experiment, with low stimulus durations the same vertical error preponderance occurred when error rates varied from $10 \%$ to over $50 \%$. The fixed horizontal bar used by Garner and Haun allowed another interpretation: More energy was required to encode correctly the higher information square, which varied vertically, than the single vertical bar, which varied horizontally. Reducing input energy could thus have resulted in more vertical dimension errors. However, with only the square varying along both dimensions in the present fixed-stem condition, the same result occurred, lessening the likelihood of that alternative explanation for the earlier finding.

The vertical error preponderance substantially disappeared when the fixation point was shifted $12 \min 9 \mathrm{sec}$ to the center of the square (the fixed-square condition). In this condition information sufficient for both horizontal and vertical discriminations was able to enter the retina equidistant from fixation. With no retinal acuity difference thus favoring one dimension, there was no asymmetry of errors between the dimensions overall.
Two of the six subjects did show a clear 2:1 vertical error preponderance in the fixed-square condition, however. This result suggests that a nonretinal mechanism may be involved. One obvious way to distinguish left or right in the fixed-square condition (as indeed in the fixed-stem and fixed-bar conditions) is the amount of stimulus left or right of fixation. Fewer horizontal errors might have resulted from the use (by these two subjects) of visual field asymmetry as a distinguishing mechanism for this one dimension. With this possibility removed by variable letter positioning (the fully variable condition), numbers of vertical and horizontal errors were virtually identical, with no subject showing a vertical-horizontal error ratio greater than $1.3: 1$. When retinal distance is equivalent for the two dimensions, then, some subjects may use a more central mechanism that produces the same error distribution as the peripheral mechanism.

The fully variable condition also demonstrated that the retinal acuity difference was not a mandatory mechanism. In this condition, the positions of the letter subset with stems at fixation (center stem) were identical to the same letters' positions in the fixed-stem condition. However, unlike in that condition, no more vertical than horizontal errors occurred. Further, a strict retinal difference interpretation predicts very few errors when the stem is left or right, compared to the center-stem subset, since both horizontal and vertical information is present immediately adjacent to fixation. The obtained result, however, showed nearly equal errors for the two subsets. When position in visual space is variable, then, retinal influence on processing is less than when position is fixed. However, as Eriksen and Schultz (1977) have shown using a reaction time measure, some retinal influence is still evident with positional variability.

The overall error distribution obtained in the fully variable condition is that expected in a stimulus set defined by two equivalent dimensions: equal numbers of confusions for stimuli differing along each dimension alone (horizontal and vertical errors), and significantly fewer confusions for stimuli differing along both dimensions (diagonal errors).

\section{REFERENCES}

Eriksen, C. W., \& Schultz, D. W. Retinal locus and acuity in visual information processing. Bulletin of the Psychonomic Society, 1977, 9, 81-84.

GARNER, W. R., \& HAUN, F. Letter identification as a function of type of perceptual limitation and type of attribute. Journal of Experimental Psychology: Human Perception and Performance, in press.

(Received for publication December 7, 1977.) 